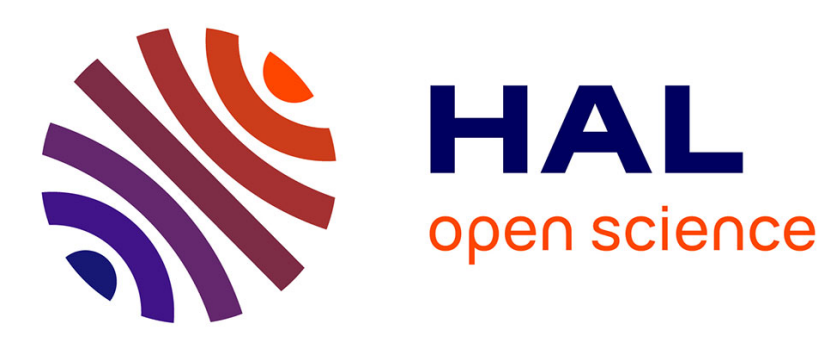

\title{
Application of Discontinuous Galerkin spectral method on hexahedral elements for aeroacoustic
}

Nicolas Castel, Gary Cohen, Marc Duruflé

\section{To cite this version:}

Nicolas Castel, Gary Cohen, Marc Duruflé. Application of Discontinuous Galerkin spectral method on hexahedral elements for aeroacoustic. Journal of Computational Acoustics, 2009, 17 (2), pp.175-196. hal-00403787

\section{HAL Id: hal-00403787 \\ https://hal.science/hal-00403787}

Submitted on 13 Jul 2009

HAL is a multi-disciplinary open access archive for the deposit and dissemination of scientific research documents, whether they are published or not. The documents may come from teaching and research institutions in France or abroad, or from public or private research centers.
L'archive ouverte pluridisciplinaire HAL, est destinée au dépôt et à la diffusion de documents scientifiques de niveau recherche, publiés ou non, émanant des établissements d'enseignement et de recherche français ou étrangers, des laboratoires publics ou privés. 


\title{
Application of Discontinuous Galerkin spectral method on hexahedral elements for aeroacoustic
}

\author{
N. CASTEL \\ INRIA Rocquencourt, projet POEMS \\ 78153 Le Chesnay, FRANCE \\ nicolas.castel@inria.fr \\ G. COHEN \\ INRIA Rocquencourt, projet POEMS \\ 78153 Le Chesnay, FRANCE \\ gary.cohen@inria.fr \\ M. DURUFLÉ \\ INRIA Rocquencourt, projet POEMS \\ 78153 Le Chesnay, FRANCE \\ marc.durufle@inria.fr \\ Received (Day Month Year) \\ Revised (Day Month Year)
}

\begin{abstract}
A discontinuous Galerkin method is developed for linear hyperbolic systems on general hexahedral meshes. The use of hexahedral elements and tensorized quadrature formulas to evaluate the integrals leads to an efficient matrix-vector product. It is shown for high order approximations, the reduction in computational time can be very important, compared to tetrahedral elements. Two choices of quadrature points are considered, the Gauss points or Gauss-Lobatto points. The method is applied to the aeroacoustic system ("simplified" Linearized Euler Equations). Some 3-D numericals experiments show the importance of penalization, and the advantage of using high order.
\end{abstract}

Keywords: Aeroacoustic, Discontinuous Galerkin, Finite Element Method, Mass Lumping, Linearized Euler Equations

\section{Discontinuous Galerkin method on hexahedral meshes}

The discontinuous Galerkin method is widely used for many applications, like Maxwell equations [3], or Linearized Euler Equations (LEE) [16]. The discontinuous Galerkin method is well suited for equations, for which the functional spaces, where the solution lies, are difficult to discretize. The use of discontinuous Galerkin method has been widely detailed in the case of tetrahedral elements [3], but less so for hexahedral elements. Cohen's article [15] describes the use of hexahedral elements in the particular case of Maxwell equations. This paper describes the implementation of the discontinuous Galerkin method on general hyperbolic systems, for hexahedral elements with mass lumping techniques, and for application to Linearized Euler Equations (LEE). A comparison of the computational efficiency between tetrahedral and hexahedral elements is done in the particular case of aeroacoustic equations 
(LEE). The advantage of using hexahedral elements is shown for high order approximations. Because of the discontinuity of the space, there are several possible quadrature formulas to perform mass lumping. Among these possiblities, two are relevant : Gauss Legendre points, and Gauss-Lobatto points. The Gauss Legendre formulas provide the best accuracy (exact integration of polynoms in $Q_{2 r+1}$ ), while the Gauss-Lobatto formulas are a bit less accurate (exact integration of polynoms in $Q_{2 r-1}$ ), but have the advantage to include the two extremities of the interval $[0,1]$, therefore leading to faster computations. For the application of the method to LEE, we will show which choice is the more efficient and robust. If nodal finite element is chosen, some spurious waves appear, and can be removed with some specific methods, as detailed in this paper solving the Galbrun equation [1]. Such methods are a bit tedious to implement, particularly on corners (cf. [2]), the exploitation of discontinuous Galerkin would seem a better alternative. Similar to the treatment for Maxwell's equations [13], we show for LEE the importance of adding "penalty" terms to the original discontinuous formulation, in order to avoid spurious waves. These penalty terms can be viewed as considering upwind fluxes instead of centered fluxes.

Let $\mathbb{R}$ denote the set of all real numbers, then

$$
\mathbb{R}^{d}=\left\{\left(x_{1}, \cdots, x_{d}\right) \text { where } x_{1}, \cdots, x_{d} \in \mathbb{R}\right\}
$$

Let us consider a linear hyperbolic system to solve in $(x, t) \in \mathbb{R}^{d} \times \mathbb{R}^{+}$:

$$
\begin{aligned}
& \left\{\begin{array}{l}
M(x) \frac{\partial U}{\partial t}+\sum_{i=1}^{d} \frac{\partial\left(A_{i}(x) U\right)}{\partial x_{i}}=F(x, t) \\
U(x, 0)=U_{0}(x)
\end{array}\right. \\
& \left\{\begin{array}{l}
A_{i}(x): R^{d} \rightarrow M_{m}(R), \forall i=1 . . d \\
F: R^{d} \times R^{+} \rightarrow R^{m}, U_{0}: R^{d} \rightarrow R^{m}
\end{array}\right.
\end{aligned}
$$

The system is hyperbolic in Friedrich's sense if

- $M(x)$ is symmetric and definite positive, - $A_{1}, \cdots, A_{d}$ are symmetric matrices for all values of the space variable $x$.

The original Linearized Euler Equations (LEE) may exhibit instabilities for the continuous equations. These instabilities are physical (called Kelvin-Helmholtz instabilities) and are limited by non-linear terms for original Euler equations, whereas they lead to exponential growths for the LEE. In [16], a special treatment is proposed to remove these instabilities, and it is compared to the model of Bailly-Bogey-Juve [11]. That model has been numerically and theoretically proven accurate, is stable, and is particularly simple to use, which is why we have chosen the Bailly-Bogey-Juve model. In this model, the LEE are 
written by introducing a "right-hand side" called $H$ whose expression is

$$
H=\mid \begin{aligned}
& (\gamma-1)\left(p \nabla \cdot v_{0}-\vec{v} \cdot \nabla p_{0}\right) \\
& \left(\rho_{0} \vec{v}+\rho v_{0}\right) \cdot \nabla v_{x} \\
& \left(\rho_{0} \vec{v}+\rho v_{0}\right) \cdot \nabla v_{y} \\
& \left(\rho_{0} \vec{v}+\rho v_{0}\right) \cdot \nabla v_{z}
\end{aligned}
$$

The LEE are then simplified by neglecting this term, leading to "simplified" LEE. As a consequence, Kelvin-Helmholtz instabilities are removed and a decoupling of the mass density $\rho$ and the pressure $p$ is performed. As a consequence, we can discretize only four unknowns (in 3-D) : the pressure $p$ and the vector $w=\rho_{0} v$. The equations associated to this model, for a flow $\vec{v}_{0}$, can be written in 2-D as an hyperbolic system as follows :

$$
\begin{aligned}
& \left\{\begin{array}{l}
\frac{\partial}{\partial t} p+\vec{v}_{0} \cdot \nabla p+c_{0}^{2} \nabla \cdot(\vec{w})=f \\
\frac{\partial}{\partial t} \vec{w}+\left(\begin{array}{l}
\vec{v}_{0} \cdot \nabla w_{x} \\
\vec{v}_{0} \cdot \nabla w_{y}
\end{array}\right)+\nabla p=\overrightarrow{0}
\end{array}\right. \\
& \Longleftrightarrow \\
& \partial_{t}\left(\begin{array}{c}
p \\
w_{x} \\
w_{y}
\end{array}\right)+\left(\begin{array}{ccc}
v_{0 x} & c_{0}^{2} & 0 \\
1 & v_{0 x} & 0 \\
0 & 0 & v_{0 x}
\end{array}\right) \partial_{x}\left(\begin{array}{c}
p \\
w_{x} \\
w_{y}
\end{array}\right)+\left(\begin{array}{ccc}
v_{0 y} & 0 & c_{0}^{2} \\
0 & v_{0 y} & 0 \\
1 & 0 & v_{0 y}
\end{array}\right) \partial_{y}\left(\begin{array}{c}
p \\
w_{x} \\
w_{y}
\end{array}\right)=\left(\begin{array}{l}
f \\
0 \\
0
\end{array}\right) \\
& \Longleftrightarrow \\
& \partial_{t} U+A_{1} \partial_{x_{1}} U+A_{2} \partial_{x_{2}} U=F
\end{aligned}
$$

where $c_{0}^{2}=\frac{\gamma \rho_{0}}{p_{0}}$.

\subsection{Variational formulation}

The Discontinuous Galerkin formulation is written on each element $K$ of the mesh as

$$
\begin{array}{r}
\int_{K} \frac{\partial u}{\partial t} \varphi d x-\sum_{i=1}^{d} \int_{K} A_{i}(x) u \\
\frac{\partial \varphi}{\partial x_{i}} d x+\int_{\partial K} \sum_{i=1}^{d} n_{i} A_{i}(x)\{u\} \varphi d x \\
+\alpha \int_{\partial K} C(x)[u] \varphi d x=\int_{K} f \varphi d x
\end{array}
$$

where $n_{i}$ is the $\mathrm{i}$-th component of the outward normal to the boundary, $\{u\}$ is the average value, $[u]$ the half the difference of $u$ across the boundary, i.e.

$$
\begin{aligned}
& \{u\}=\frac{1}{2}\left(u_{1}+u_{2}\right) \\
& {[u]=\frac{1}{2}\left(u_{2}-u_{1}\right)}
\end{aligned}
$$


where $u_{1}$ and $u_{2}$ are the two values of $u$ across the boundary. $u_{1}$ is the "inside" value defined on the element considered $K$.

The computational domain $\Omega$ is discretized with a mesh of quadrilateral/hexahedral elements :

$$
\Omega=\bigcup_{e} K_{e}
$$

A quadrilateral/hexahedral element is defined as the image, by the classical bilinear/trilinear map $F_{e}$, of the unit square/cube $\hat{K}=[0,1]^{d}$. An illustration of the transformation $F_{e}$ is shown in Fig 1 . We denote $D F_{e}$ as Jacobian matrix of this transformation, and $J_{e}$ its deter-

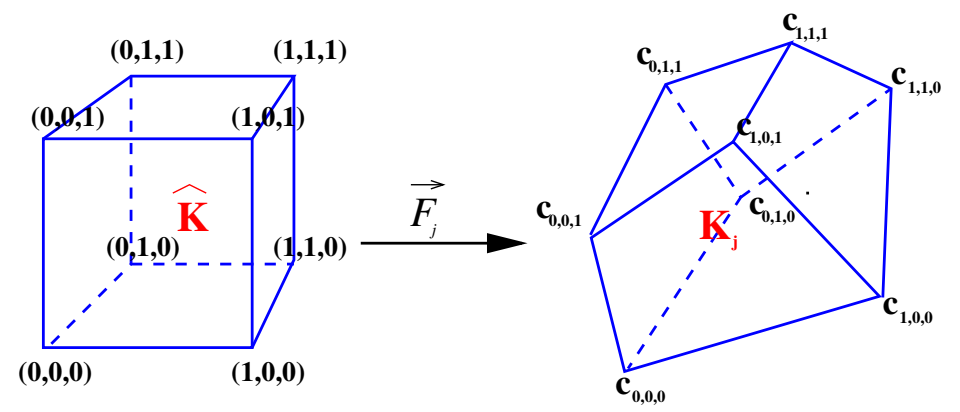

Fig. 1. $F_{e}$ for the dimension 3.

minant. A more detailed definition of the properties required by this trilinear transformation in order to have a non-degenerated element, can be found in [7]. The finite element space is

$$
U_{h}^{r}=\left\{u \in L^{2}(\Omega) \quad \forall e,\left.\quad u\right|_{K_{e}} \in Q_{r}\right\}
$$

where $Q_{r}$ is the space of polynomials whose degree is equal or less than $r$ in each variable.

For the discretization of $Q_{r}$, the use of tensorized basis functions will take advantage of the tensorized structure of the cube. Our choice has been to use a "mass-lumping" technique [14], in order to get a diagonal mass matrix, and to deal easily with the case where the coefficients $A_{i}$ vary pointwise inside each element. The mass lumping technique selects the same points for both quadrature formulas and degrees of freedom (Lagrange interpolation). We shall consider the Gauss-Legendre or Gauss-Lobatto points and related quadrature formulas, as described in [8] or [9]. These are obtained by tensor products of 1-D points, as shown in Fig 2. Below is the fundamental accuracy property of the Gauss and Gauss-Lobatto quadrature rules 


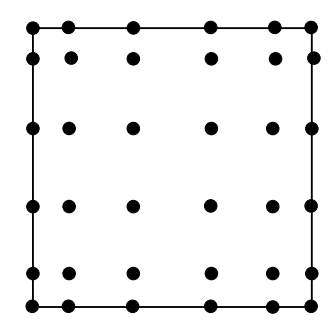

Fig. 2. 2-D Gauss-Lobatto points for $r=5$

Proposition 1.1. If we denote by $\left(\widehat{\omega}_{i}^{G}, \widehat{\xi}_{i}^{G}\right)$ and $\left(\widehat{\omega}_{i}^{G L}, \widehat{\xi}_{i}^{G L}\right), 1 \leq i \leq(r+1)^{d}$, the Gauss and Gauss-Lobatto weights and points in $\widehat{K}$ [8], then we have:

$$
\begin{gathered}
\int_{\widehat{K}} \widehat{f}(\widehat{x}) d \widehat{x}=\sum_{k=1}^{(r+1)^{d}} \widehat{\omega}_{k}^{G} \widehat{f}\left(\widehat{\xi}_{k}^{G}\right), \quad \forall \widehat{f} \in Q_{2 r+1}(\widehat{K}), \\
\int_{\widehat{K}} \widehat{g}(\widehat{x}) d \widehat{x}=\sum_{k=1}^{(r+1)^{d}} \widehat{\omega}_{k}^{G L} \widehat{g}\left(\widehat{\xi}_{k}^{G L}\right), \quad \forall \widehat{g} \in Q_{2 r-1}(\widehat{K}) .
\end{gathered}
$$

The basis functions based on these degrees of freedom are tensorized as follows

$$
\hat{\varphi}_{i}\left(\hat{x}_{1}, \hat{x}_{2}, \hat{x}_{3}\right)=\hat{\varphi}_{i_{1}}\left(\hat{x}_{1}\right) \hat{\varphi}_{i_{2}}\left(\hat{x}_{2}\right) \hat{\varphi}_{i_{3}}\left(\hat{z}_{3}\right)
$$

For each " 3 -D" index $i$, there is a corresponding tuple denoted $\left(i_{1}, i_{2}, i_{3}\right)$. The basis functions on the hexahedron $K_{e}$ are defined via the transformation $F_{e}$ :

$$
\varphi_{i} \circ F_{e}=\hat{\varphi}_{i}
$$

The problem is to find $u_{h} \in U_{h}^{r}$ so that

$$
\left\{\begin{array}{l}
\forall K \in \Omega_{h}, \quad \forall \varphi_{h} \in U_{h}^{r} \\
\int_{K} \frac{\partial u_{h}}{\partial t} \varphi_{h} d x-\sum_{i=1}^{d} \int_{K} A_{i}(x) u_{h} \frac{\partial \varphi_{h}}{\partial x_{i}} d x+\int_{\partial K} \sum_{i=1}^{d} n_{i} A_{i}(x)\left\{u_{h}\right\} \varphi_{h} d x \\
+\int_{\partial K} C(x)\left[u_{h}\right] \varphi_{h} d x=\int_{K} f \varphi_{h} d x
\end{array}\right.
$$

We define the mass matrix

$$
\left(M_{h}\right)_{j, k}=\sum_{e} \int_{K_{e}} \varphi_{j} \varphi_{k} d x
$$

the stiffness matrix

$$
\left(K_{h}\right)_{j, k}=-\sum_{e}\left(\sum_{i=1}^{d} \int_{K_{e}} A_{i}(x) \varphi_{k} \frac{\partial \varphi_{j}}{\partial x_{i}} d x+\int_{\partial K_{e}} \sum_{i=1}^{d} n_{i} A_{i}(x)\left\{\varphi_{k}\right\} \varphi_{j} d x\right)
$$


and the penalty matrix

$$
\left(P_{h}\right)_{j, k}=\sum_{e} \int_{\partial K_{e}} C(x)\left[\varphi_{k}\right] \varphi_{j} d x
$$

We then have the following differential system :

$$
M_{h} \frac{\mathrm{d} U}{\mathrm{~d} t}+K_{h} U+\alpha P_{h} U=F_{h}(t)
$$

In the case of a symmetric system (ie the matrices $A_{i}$ are symmetric), we can split the matrices $A_{i}$ as follows

$$
A_{i}=B_{i}+B_{i}^{*}
$$

Several splittings are possible, we propose to use the following one :

$$
B_{i}=\frac{1}{2} D_{i}+L_{i}
$$

where $D_{i}$ is the diagonal matrix of $A_{i}$ and $L_{i}$ the lower triangular part (not including diagonal). In the variational formulation, we do integration by parts on one term, so that we obtain the "split" formulation

$$
\begin{aligned}
\int_{K} \frac{\partial u}{\partial t} \varphi d x & -\sum_{i=1}^{d} \int_{K} B_{i}(x) u \frac{\partial \varphi}{\partial x_{i}} d x+\int_{\delta K} \sum_{i=1}^{d} n_{i} B_{i}(x)\{u\} \varphi d x \\
& +\sum_{i=1}^{d} \int_{K} B_{i}^{*}(x) \frac{\partial u}{\partial x_{i}} \varphi d x+\int_{\delta K} \sum_{i=1}^{d} n_{i} B_{i}^{*}(x)[u] \varphi d x \\
& +\int_{K} \frac{\partial B_{i}}{\partial x_{i}} u \varphi d x+\int_{\delta K} \sum_{i=1}^{d} C_{i}(x)[u] \varphi d x=\int_{K} f \varphi d x
\end{aligned}
$$

The advantage of this decomposition is to explicitely show the skew-symmetry of the stiffness matrix $K_{h}$, in the special case where the matrices $A_{i}$ are independent of the location (the term $\frac{\partial A_{i}}{\partial x_{i}}$ is equal to 0 ), and the penalty terms are set to zero.

The disadvantage of this split formulation is slower matrix-vector calculations. Hence, we have not used this formulation in practice and will provide more detail in the next subsection.

\subsection{Stability}

The skew-symmetry of the stiffness matrix induces a conservative scheme, if using a secondorder leap-frog scheme [4]. In this thesis, the author shows the conservation of an energy and thus the stability of the scheme. This study is done on tetrahedral elements and can easily be extended to hexahedral elements since the only property needed is the skew-symmetry of the matrix. 
Theorem 1.1. In the case where the matrices $A_{i}$ are uniform per element, a quadrature formula exact for $Q_{2 r+1}$ is exact for the evaluation of the stiffness matrix

Proof. The demonstration is done in 3-D. Let us define :

$$
Q_{r_{1}, r_{2}, r_{3}}=\operatorname{span}\left[x_{1}^{\alpha_{1}} x_{2}^{\alpha_{3}} x_{d}^{\alpha_{d}}\right], \quad \alpha \in \mathcal{S}_{r_{1}, r_{2}, r_{3}}=\left\{\alpha \in \mathbb{N}^{3}, \quad \alpha_{j} \leq r_{j}, \forall j\right\} .
$$

We have the following assessment

$$
\text { if } u \in Q_{r_{1}, r_{2}, r_{3}}, v \in Q_{r_{1}^{\prime}, r_{2}^{\prime}, r_{3}^{\prime}} \text { then } u v \in Q_{r_{1}+r_{1}^{\prime}, r_{2}+r_{2}^{\prime}, r_{3}+r_{3}}
$$

This property will be extensively used to estimate the polynomial degrees of the different terms. $\hat{\nabla}$ is the gradient operator for variable $\hat{x}$ relying on the unit cube $\hat{K}$ whereas $\nabla$ is the gradient operator for variable $\hat{x}$ relying on the real hexahedron $K$. After a change of variables, the volumic integral in the stiffness matrix can be written as

$$
\sum_{i} \int_{\hat{K}} A_{i}^{p, q} \hat{\varphi}_{k}\left(J_{e} D F_{e}^{*-1} \hat{\nabla} \hat{\varphi}_{j}\right) \cdot e_{i} d x
$$

By definition, the transformation $F_{e} \in Q_{1,1,1}^{3}$. Differentiating with respect to each variable, we lose one order, thus we obtain the following properties for the Jacobian matrix :

$$
\left(D F_{e}\right)_{i, 1} \in Q_{0,1,1},\left(D F_{e}\right)_{i, 2} \in Q_{1,0,1},\left(D F_{e}\right)_{i, 3} \in Q_{1,1,0}
$$

Matrix $J_{e} D F_{e}^{*-1}$ is the transpose of the cofactors of $D F_{e}$, and a straightforward computation provides

$$
\left(J_{e} D F_{e}^{*-1}\right)_{1, i} \in Q_{2,1,1},\left(J_{e} D F_{e}^{*-1}\right)_{2, i} \in Q_{1,2,1},\left(J_{e} D F_{e}^{*-1}\right)_{3, i} \in Q_{1,1,2}
$$

For a basis function $\hat{\varphi}_{i} \in Q_{r, r, r}$, we have

$$
\hat{\nabla} \hat{\varphi}_{i} \in Q_{r-1, r, r} \times Q_{r, r-1, r} \times Q_{r, r, r-1}
$$

Finally, by summing the polynomial degrees, we get

$$
J_{e} D F_{e}^{*-1} \hat{\nabla} \hat{\varphi}_{i} \in Q_{r+1}^{3}
$$

Thus

$$
\hat{\varphi}_{k}\left(J_{e} D F_{e}^{*-1} \hat{\nabla} \hat{\varphi}_{j}\right) \cdot e_{i} \in Q_{2 r+1}
$$

For the surface integral in the stiffness matrix, the change of variables provides

$$
\sum_{i} \int_{\partial \hat{K}} d s_{e} n_{e} A_{i}^{p, q} \hat{\varphi}_{j} \hat{\varphi}_{k} d x
$$

The weighed normal $d s_{e} n_{e}$ belongs to $Q_{1}$ because each face of the hexahedron is defined as image of a bilinear transformation $F_{N}$ from the unit square. As a consequence $d s_{e} n_{e} \hat{\varphi}_{j} \hat{\varphi}_{k} \in$ $Q_{2 r+1}$. Therefore, We need a quadrature formula exact for $Q_{2 r+1}$, to exactly compute the stiffness matrix. 
The use of Gauss points leads then to an exact evaluation of the stiffness matrix (for the mass matrix, the integration is not exact in $3-\mathrm{D}$, because $J_{e} \in Q_{2}$ ). That's why we have the same stability result as for tetrahedral elements. The use of Gauss-Lobatto points provide an approximate integration, and one can prove the stability only when using the split formulation (6). If we use the original formulation (5) to compute the stiffness matrix with Gauss-Lobatto points, the computed stiffness matrix is not skew-symmetric and can lead to instabilities. Nevertheless, these instabilities can be removed by setting a penalty term large enough in the original formulation. However, the stability in that case is an open question : an explanation can be that the instability is eliminated for a sufficiently long period of time so that the instability is not observed in our experiments.

\subsubsection{Effect of penalization}

We introduced a penalization term with a matrix C. If an upwind flux is chosen (Roe flux), the matrix $C$ would read

$$
C=\left|\sum A_{i} n_{i}\right|
$$

In the case of a null flow, it is possible to prove that (if $\alpha=\delta=1$ )

$$
C=\left(\begin{array}{ll}
\alpha & 0 \\
0 & \delta n \\
n & n^{*}
\end{array}\right)
$$

with $\alpha<0$ and $\delta<0$.

We could also have taken $C=\alpha I d$ or $C$ diagonal with negative coefficients, the effects are similar. We chose to consider the same matrix $C$ even for a non-uniform flow, the main advantage is to have a matrix $C$ independent of the flow. The case where $\alpha=$ delta $=0$ corresponds to centered fluxes, whereas the case $\alpha=\delta=1$ correspond to classical Roe fluxes (upwind fluxes). For the acoustic equation (flow set to zero), the effect of penalization is to reduce the dispersion [5] and improve the order of convergence [3], but it adds some dissipation and degrades the CFL stability condition. To illustrate the benefit of the penalization in the solution, we display in Fig 3 the time-harmonic solution in an open domain (bounded with PML layers). The solution is slightly better, but the necessity of penalization is not obvious.

Let us try the same experiment with an uniform flow $M=(0.5 ; 0)$. As shown in Fig 4, the improvment is remarkable. We observe the same type of results, if absorbing, Neumann, or periodic boundary conditions are used. The penalization has the effect of removing spurious modes, as it has been observed for Maxwell equations [13]. Another beneficial effect of penalization is the possibility of using Gauss-Lobatto points without using the split formulation (6).

The need for penalization in the case of a non-null flow is obvious. So, we shall always use this penalization in the numerical experiments. For the choice of the coefficients $\alpha$ and $\delta$, 

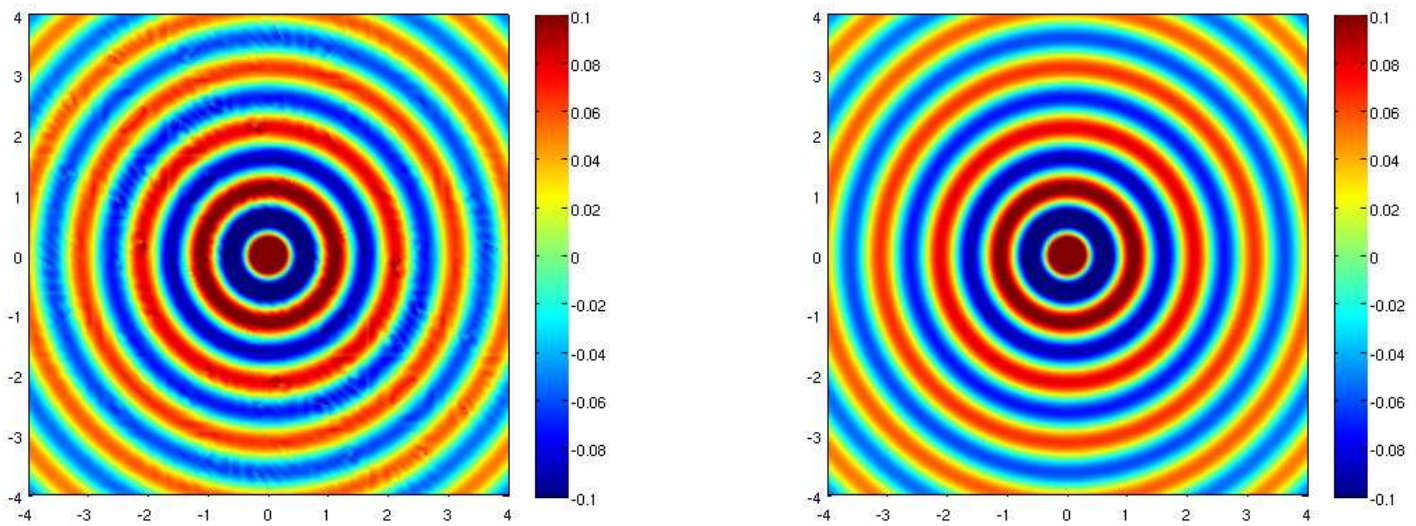

Fig. 3. Point source in an open domain with a null flow. At left, without penalization and at right with $\alpha=-0.5$. A triangular mesh split of quadrilaterals is used.
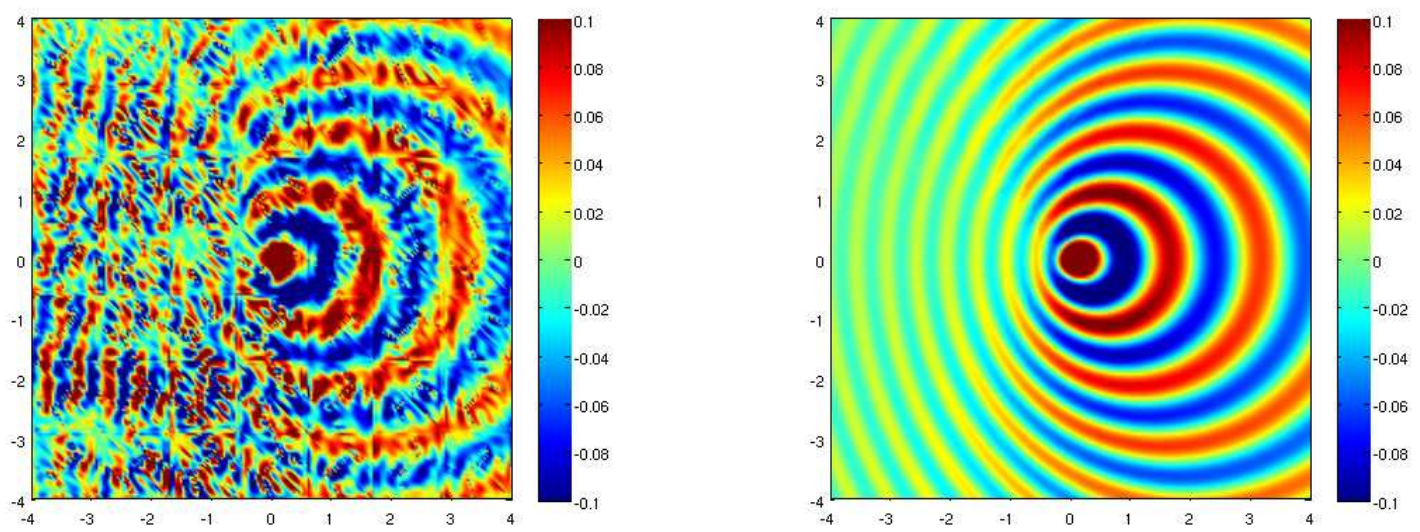

Fig. 4. Point source in an open domain with a uniform flow. At left, without penalization and at right with $\alpha=\delta=-0.5$. Triangular meshes split in quadrilaterals are used.

the error made on the solution displayed in Fig 4 is computed for different values of these parameters. In Fig 5 , we can see that the solution is dramatically improved by adding these penalization terms. For a regular mesh, the error decreases from $5 \%$ to $0.2 \%$, and for a distorted mesh, the error decreases from $35 \%$ to $1 \%$. There is no "special" optimal value of these parameters, but a good choice can be

$$
\alpha=\delta=-0.5
$$

When the mesh is refined, or if the order of approximation is modified, similar error reductions are observed. 

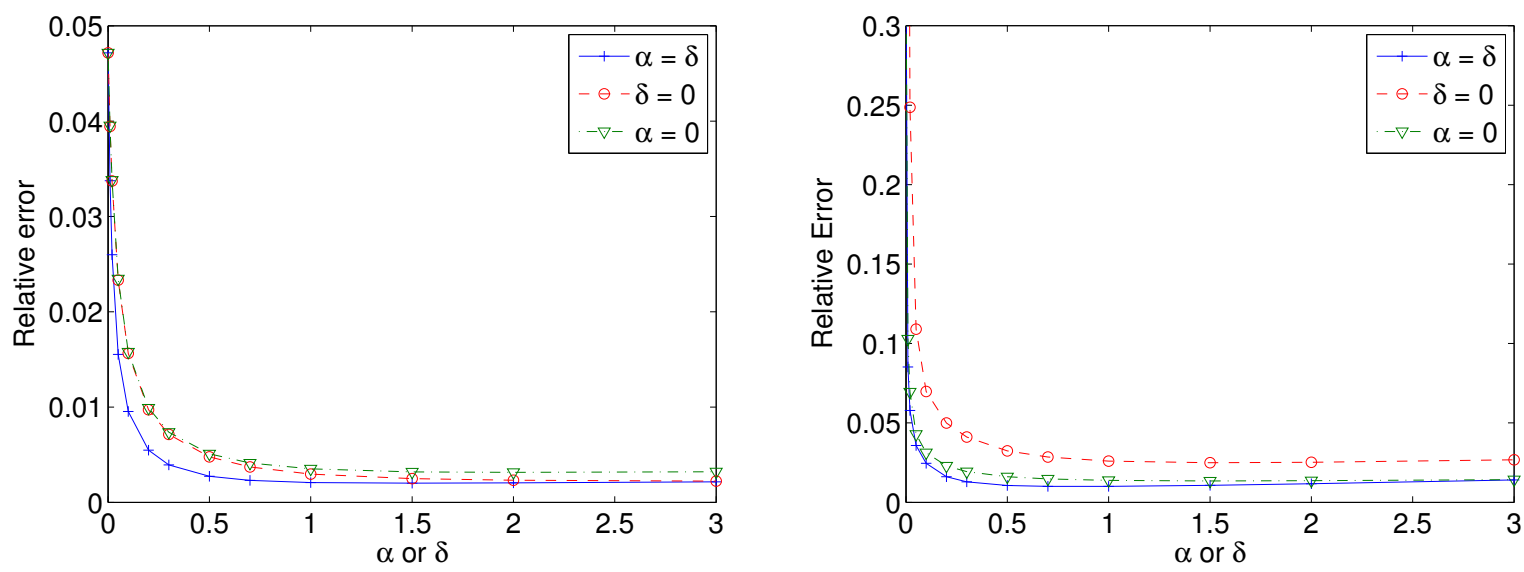

Fig. 5. Relative error according to the absolute value of $\alpha$ or $\delta$, if we choose $\alpha=\delta, \alpha=0$ or $\delta=0$. At left, the mesh is regular, at right the mesh is composed of triangles split in quads. Gauss-Lobatto points are used with a $Q_{5}$ approximation.

These penalization terms decrease the CFL number, which slows the computation. To illustrate this effect, the maximal time step for which stability is observed is displayed in Fig 6 when $\alpha=\delta$ for a Runge-Kutta scheme. In these results, the CFL doesn't decrease until the

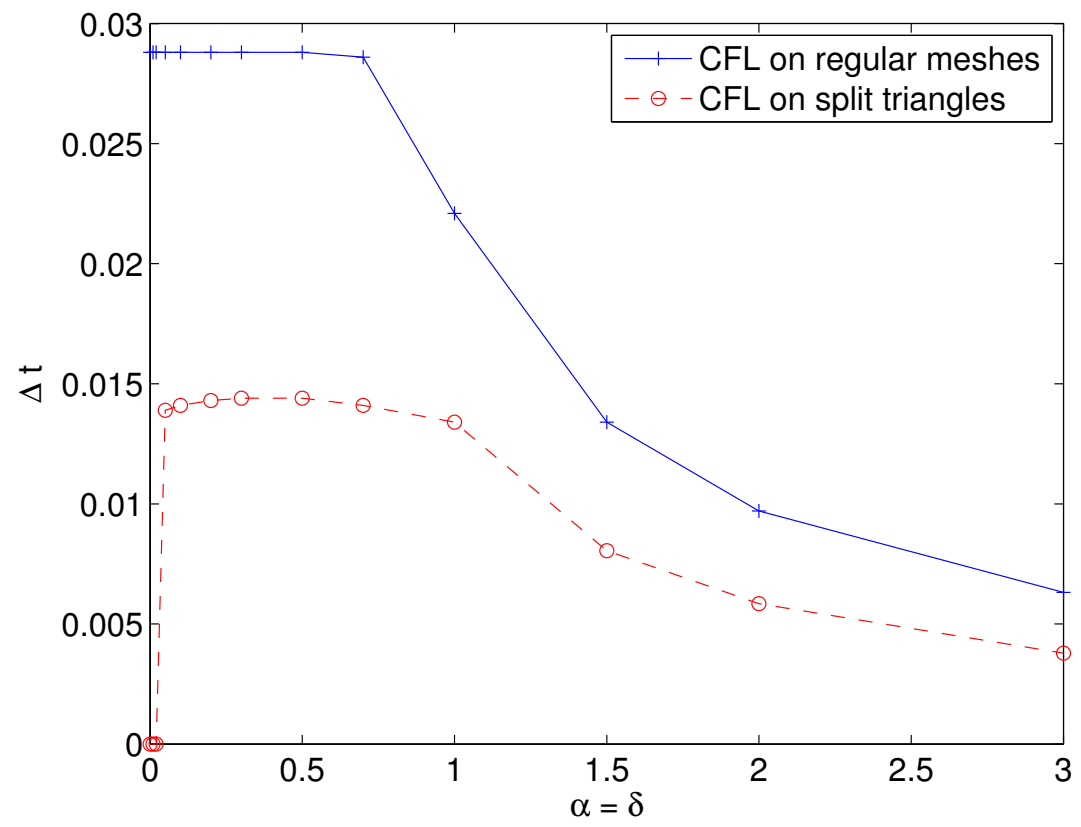

Fig. 6. Maximal $\Delta t$ for which the fourth order Runge-Kutta scheme is stable, for a regular or distorted mesh. 
value approaches

$$
\alpha=\delta=-0.5
$$

Then, the reduction scales as $1 / \alpha$. In the case of distorted mesh, the CFL observed for very small values of $\alpha$ is equal to 0 , because Gauss-Lobatto points are used with the original formulation (5). We have already mentioned that in this case, instabilities can be observed. It is important to note that these instabilities are "spatial" in the sense that they always occur at the same time $T$, independently of the time scheme and time step used. The effect of the penalization is to reduce these instabilities, i.e. the value $T$ increases with the value of $|\alpha|$, so that for $|\alpha|$ large enough, we do not observe the instability during the simulation time period. An illustration of this phenomenon is provided in Fig 7 For $\alpha=-0.5$, instability

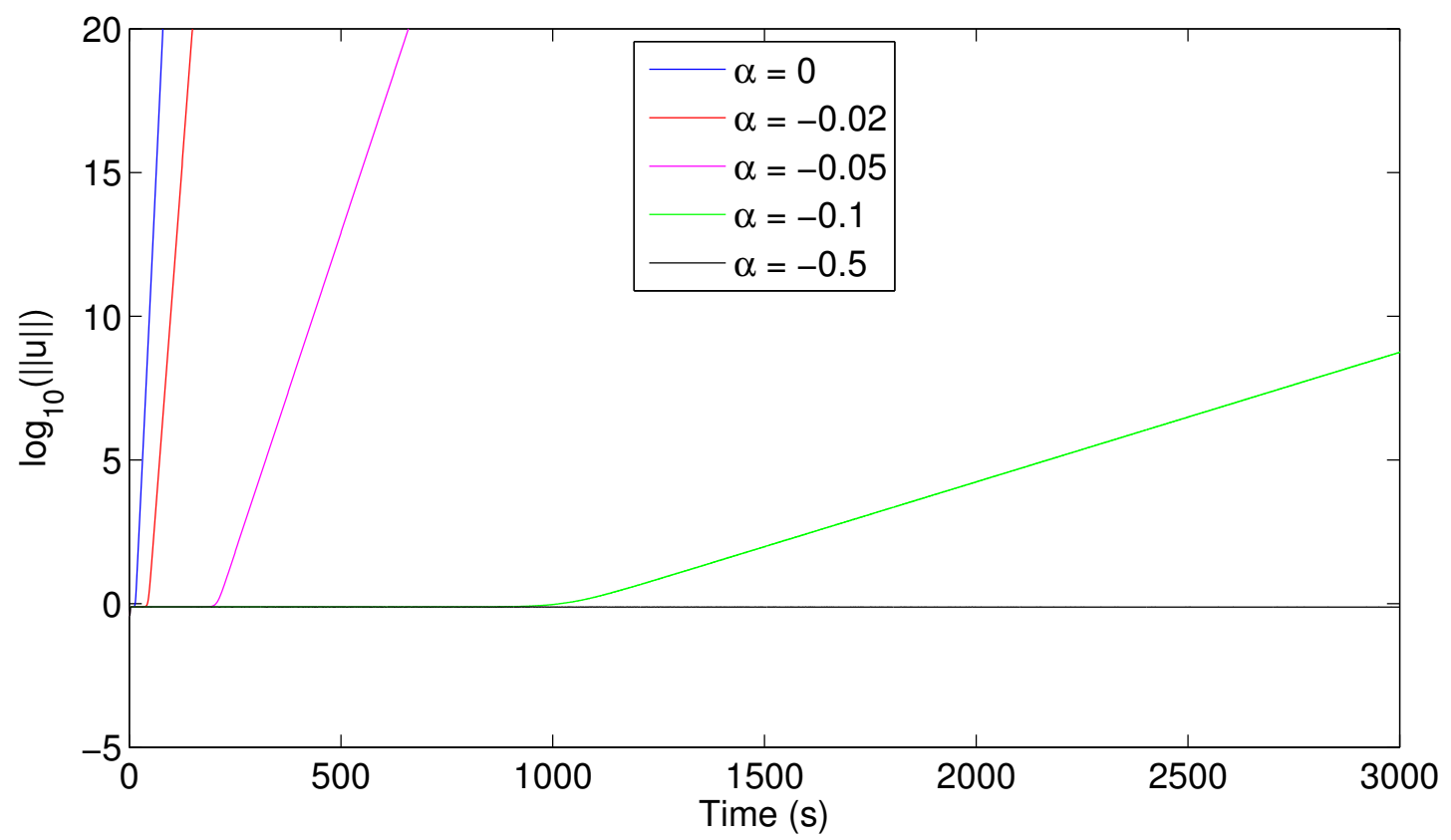

Fig. 7. Time evolution of the logarithm of the $L^{2}$ norm of the solution. Gauss-Lobatto points are used on a distorted mesh and different values of the penalization parameter are selected

was not observed until $T=100000 \mathrm{~s}$.

The behaviour of the deterioration of the CFL appears to depend on the time scheme, the mesh and the order of approximation, Let us denote $\alpha^{*}$ as the value at which the CFL begins to decrease. In Fig $6, \alpha^{*}$ is approximately -0.5 . When the mesh is refined or the order increased, $\alpha^{*}$ increases.

The effect of penalization is similar when using Gauss points instead of Gauss-Lobatto. The only difference is that Gauss points provide always stable solutions (e.g. the figure 7 is not observed for Gauss points) as proven theoretically in a previous theorem. 


\subsection{Fast matrix-vector product}

Let us denote $n$ the number of unknowns (4 for the 3-D aeroacoustic equations), $N_{\text {dof }}$ the number of degrees of freedom on an hexahedron $\left(N_{\text {dof }}=(r+1)^{3}\right)$ The row index is denoted $(p, e, j)$ where $e$ is the number of element and $j$ the local number of degree of freedom inside the element, $p$ the number of the unknown. The column index is denoted $\left(q, e^{\prime}, k\right)$.

\subsubsection{Mass matrix}

Because of mass-lumping, the mass matrix is diagonal and we have

$$
\left(M_{h}\right)_{(p, e, j),\left(q, e^{\prime}, k\right)}=\delta_{p, q} \delta_{e, e^{\prime}} \delta_{j, k} J_{e}\left(\xi_{k}\right) \omega_{k}
$$

\subsubsection{Stiffness matrix}

We can split the stiffness matrix in two parts: $K_{h}=R_{h}+S_{h}$

The volumic integral component in the stiffness matrix is:

$$
\begin{aligned}
\left(R_{h}\right)_{(p, e, j),\left(q, e^{\prime}, k\right)} & =\delta_{e, e^{\prime}} \int_{K_{e}} \sum_{i} A_{i}^{p, q} \varphi_{k} \frac{\partial \varphi_{j}}{\partial x_{i}} d x \\
& \approx \delta_{e, e^{\prime}} \sum_{i} \omega_{k}\left(J_{e} A_{i}^{p, q}\right)\left(\hat{\xi}_{k}\right) \frac{\partial \varphi_{j}}{\partial x_{i}}\left(\xi_{k}\right) \\
& =\delta_{e, e^{\prime}} \sum_{i, m} \omega_{k}\left[\left(J_{e} D F_{e}^{-1}\right)_{m, i} A_{i}^{p, q}\right]\left(\hat{\xi}_{k}\right) \frac{\partial \hat{\varphi}_{j}}{\partial \hat{x}_{m}}\left(\hat{\xi}_{k}\right)
\end{aligned}
$$

So, we choose to store only the $d \times d$ matrices $J_{e} D F_{e}^{-1}$, i.e. the values of $J_{e} D F_{e}^{-1}$ at each degree of freedom. The matrix-vector product $Y=R_{h} U$ is calculated "on the fly", for each element, in three steps :

(1) $v_{i, p, k}=\sum_{q=1}^{n} A_{i}^{p, q} u_{k}^{q}, \quad i=1 . . d, p=1 . . n, k=1 . . N_{\mathrm{dof}}$

(2) $w_{m, p, k}=\omega_{k} \sum_{i=1}^{d}\left(J_{e} D F_{e}^{-1}\right)_{m, i}\left(\hat{\xi}_{k}\right) v_{i, p, k}, \quad m=1 . . d, p=1 . . n, k=1 . . N_{\mathrm{dof}}$

(3) $y_{p, j}=\sum_{m=1}^{d} \sum_{k=1}^{N d o f} \frac{\partial \hat{\varphi}_{j}}{\partial \hat{x}_{m}}\left(\hat{\xi}_{k}\right) w_{m, p, k}, \quad p=1 . . n, j=1 . . N_{\mathrm{dof}}$

The first step applies the matrices $A_{i}$, the second step applies geometrical transformations and the final step performs the integration against derivatives of basis functions.

These steps can be seen as a factorization of matrix $R_{h}$ as follows

$$
R_{h}=\widehat{S} D F_{h} A_{h}
$$

The multiplication by the matrices $A_{h}, D F_{h}$ and $\widehat{S}$ corresponds to the first, second and third step of the previous algorithm. Matrix $D F_{h}$ is a $d \times d$ block-diagonal matrix.

The calculational scaling of the two first steps is obviously in $O\left(r^{3}\right)$ whereas the last step 
seems to scale as $O\left(r^{6}\right)$ because of the loop in $N_{\text {dof }}$. But that order is too high because matrix $\widehat{S}$ is very sparse on hexahedral elements due to the tensorization of the degrees of freedom. To compute each derivative, only $r+1$ values are needed, and not $(r+1)^{3}$ values. To illustrate this property, we decompose each index into its three coordinates :

$$
j=\left(j_{1}, j_{2}, j_{3}\right), \quad k=\left(k_{1}, k_{2}, k_{3}\right)
$$

The basis functions are tensorized as follows

$$
\hat{\varphi}_{j}\left(\hat{x}_{1}, \hat{x}_{2}, \hat{x}_{3}\right)=\hat{\varphi}_{j_{1}}\left(\hat{x}_{1}\right) \hat{\varphi}_{j_{2}}\left(\hat{x}_{2}\right) \hat{\varphi}_{j_{3}}\left(\hat{x}_{3}\right)
$$

The first term of the last step can be then written as

$$
y_{p, j}=\sum_{k_{1}, k_{2}, k_{3}=1}^{r+1} \frac{\partial \hat{\varphi}_{j_{1}}}{\partial \hat{x}_{1}}\left(\hat{\xi}_{k_{1}}\right) \hat{\varphi}_{j_{2}}\left(\hat{\xi}_{k_{2}}\right) \hat{\varphi}_{j_{3}}\left(\hat{\xi}_{k_{3}}\right) w_{1, p, k}
$$

The basis functions are Lagrange functions with the fundamental property :

$$
\hat{\varphi}_{i}\left(\hat{\xi}_{j}\right)=\delta_{i, j}
$$

Thus, the triple sum is reduced to a single sum :

$$
y_{p, j}=\sum_{k_{1}=1}^{r+1} \frac{\partial \hat{\varphi}_{j_{1}}}{\partial \hat{x}_{1}}\left(\hat{\xi}_{k_{1}}\right) w_{1, p,\left(k_{1}, j_{2}, j_{3}\right)}
$$

We clearly see that we need only $r+1$ terms to compute the derivative along $x_{1}$. Similar computations can be done for the derivatives along $x_{2}$ and $x_{3}$.

\subsection{3. "Jump" matrix}

We now show the method to calculate the "jump" matrix, i.e. matrix coming from boundary integrals of the variational formulation. For the sake of simplicity, we write only the "internal" part of the jump matrix (and not interactions between the elements).

$$
S_{h(p, e, j),\left(q, e^{\prime}, k\right)}=\delta_{e, e^{\prime}} \int_{\partial \hat{K}} d s_{e} \sum_{i} n_{i} A_{i}^{p, q} \hat{\varphi}_{k} \hat{\varphi}_{j} d x
$$

where $d s_{e}$ is the length of an edge in $2 D$ or the surface of a face in $3 D$.

After integration over quadrature points $\hat{\zeta}_{m}$ on the boundary, the matrix-vector product can be written as

$$
\left(S_{h} U\right)_{p, j}=\sum_{m, q, k, i} \omega_{m}\left(n_{i} d s_{e} A_{i}^{p, q}\right)\left(\hat{\zeta}_{m}\right) \hat{\varphi}_{k}\left(\hat{\zeta}_{m}\right) \hat{\varphi}_{j}\left(\hat{\zeta}_{m}\right) u_{k}^{q}
$$

This can be split into three steps

(1) $v_{q, m}=\sum_{k} \hat{\varphi}_{k}\left(\hat{\zeta}_{m}\right) u_{k}^{q}$ 
(2)

$$
\begin{aligned}
& \text { (2) } w_{p, m}=\omega_{m} \sum_{q, i}\left(n_{i} d s_{e} A_{i}^{p, q}\right)\left(\hat{\zeta}_{m}\right) v_{q, m} \\
& \text { (3) } y_{p, j}=\sum_{m} \hat{\varphi}_{j}\left(\hat{\zeta}_{m}\right) w_{p, m}
\end{aligned}
$$

This also can be seen as the following factorization

$$
S_{h}=\widehat{E} N_{h} \widehat{E}^{*}
$$

Matrix $\widehat{E}$ computes the extrapolation needed to determine the value of $U$ on quadrature points on edges.

When we use Gauss-Lobatto points, matrix $\widehat{E}$ is the identity matrix, because the GaussLobatto points contain the two ends of the interval $[0,1]$. Thus, we have the values on edges. In the case of Gauss points, we need to know the values on Gauss points on edges/faces. By using hexahedral mesh, matrix $\widehat{E}$ is very sparse because of the tensorization of the degrees of freedom.

In Fig 8, an example is shown for $Q_{2}$.

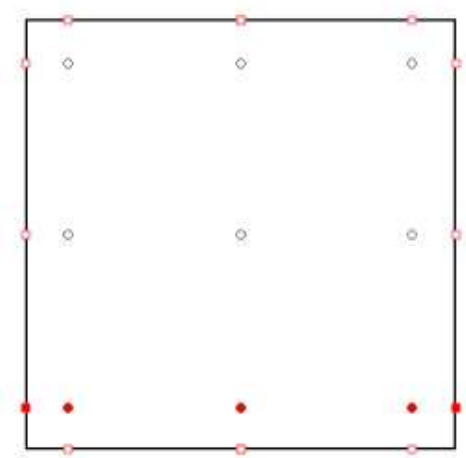

Fig. 8. Degrees of freedom necessary to compute extrapolation on the quadrature points of the edge.

\subsection{Computational complexity}

In order to compare hexahedral elements to tetrahedral elements, we show computational scaling. In the case of tetrahedral elements, one can write similar algorithms by using any type of interpolation points (it works only for straight elements, i.e. without curvature), without the mass lumping technique [3]. Again the notations are $d$ the dimension, $r$ the order of approximation, $n$ the number of unknowns and $N_{\text {dof }}$ the number of degrees of freedom inside an element.

For hexahedra, $N_{\text {dof }}=(r+1)^{3}$ and for tetrahedra $N_{\text {dof }}=\frac{(r+1)(r+2)(r+3)}{6}$

Matrices $A_{i}$ are considered full, whereas in most applications, these matrices are sparse and require less operations.

All the costs detailed next, are costs for ONE element of the mesh. 
Cost of the product by mass matrix : $N_{\text {dof }} n$ operations

Cost of the product by $R_{h}$ for hexahedral elements : $2 d n^{2} N_{\text {dof }}+2 d^{2} n N_{\text {dof }}+$ $2 d n N_{\text {dof }}(r+1)$

Cost of the product by $R_{h}$ for tetrahedral elements : $2 d n^{2} N_{\text {dof }}+2 d^{2} n N_{\text {dof }}+2 d n N_{\text {dof }}^{2}$ Cost of the product by $S_{h}$ for hexahedral elements (Gauss points) : $24 n N_{\text {dof }}+18 d n^{2}(r+$ $1)^{2}$

Cost of the product by $S_{h}$ for hexahedral elements (GL points) : $18 d n^{2}(r+1)^{2}$

Cost of the product by $S_{h}$ for tetrahedral elements : $6 d n^{2} r(r+1)$

The main economy realized by using hexahedral elements is for the evaluation of derivatives. For each point of quadrature, only $(r+1)$ values are needed to evaluate derivative along $\mathrm{x}$. This results in a scaling of $O\left(r^{4}\right)$ (the greatest term is $2 d n N_{\text {dof }}(r+1)$, the scaling $N_{\text {dof }}$ is of $O\left(r^{3}\right)$ ). For tetrahedral elements all the $N_{\text {dof }}$ values are needed to evaluate derivatives, resulting in scaling of $O\left(r^{6}\right)$. In Fig 9, we have displayed the number of operations needed
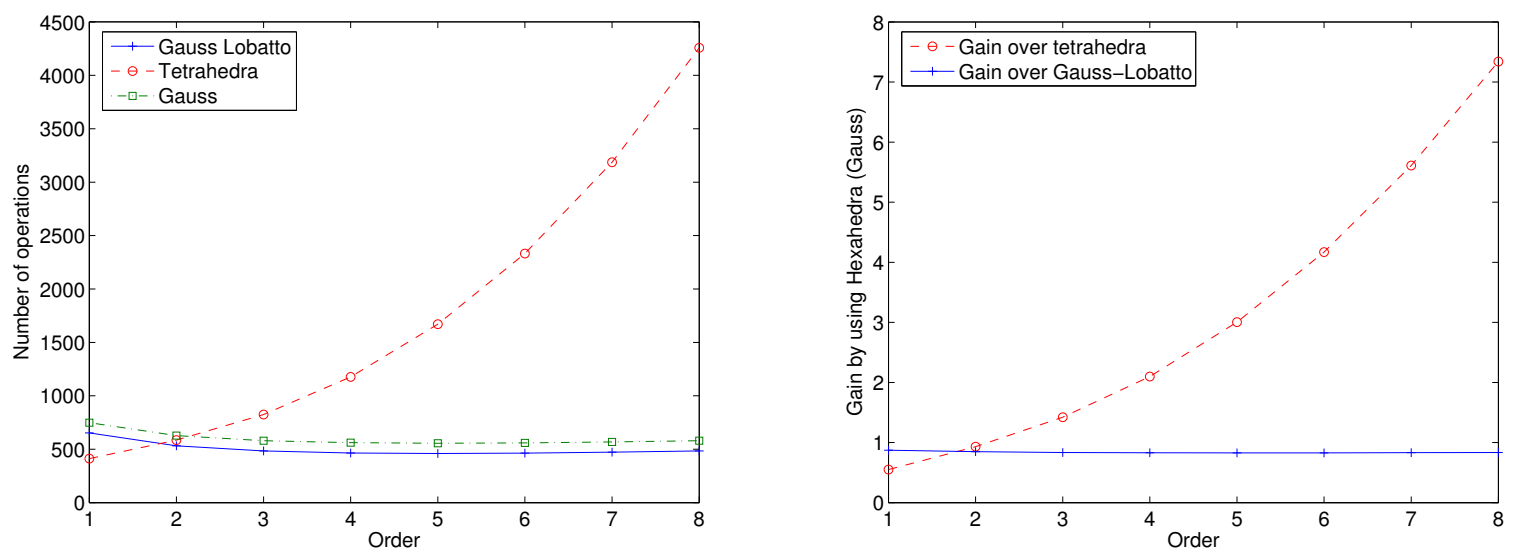

Fig. 9. Number of operations per dof for tetrahedral or hexahedral elements according the order of approximation. We selected $d=3 n=4$ (four unknowns for the aeroacoustic equations). At right, gain obtained by using Gauss points for hexahedral elements over tetrahedral elements or Gauss-Lobatto points.

by degree of freedom. The cost for hexahedral elements is almost constant versus the order of approximation, whereas it can become very large for tetrahedral elements. In particular example, for a $Q_{5}$ approximation, hexahedra are three times faster than tetrahedra.

For tetrahedral elements, the storage required is quite neglegible because the matrices $D F_{e}$ are constant inside each element, whereas for hexahedral elements we need to store the values of $D F_{e}$ on each degree of freedom, and also the normal vector on each face. It induces a storage of $9+\frac{9}{r+1}$ coefficients per degree of freedom. for the aeroacoustic system, for example $(n=4)$, this storage is equivalent between two and three vectors. The additional storage induced by the use of hexahedral elements is minimal. 


\subsubsection{Gauss or Gauss-Lobatto?}

As described in the previous section, the Gauss-Lobatto points enable a faster matrix-vector product than Gauss points.

\begin{tabular}{|c|c|c|c|c|c|c|c|}
\hline Order & $Q_{1}$ & $Q_{2}$ & $Q_{3}$ & $Q_{4}$ & $Q_{5}$ & $Q_{6}$ & $Q_{7}$ \\
\hline \hline Gauss points & 0.50000 & 0.24748 & 0.15063 & 0.10164 & 0.07319 & 0.05518 & 0.04308 \\
\hline GL points & 1.00000 & 0.39493 & 0.21966 & 0.14019 & 0.09686 & 0.07071 & 0.05377 \\
\hline
\end{tabular}

Table 1. 1-D CFL numbers for Gauss or GL points, with no penalization $(\alpha=\delta=0)$, no flow and for a leap frog scheme

Another advantage of Gauss-Lobatto points is that the CFL number is higher than that of Gauss points. The CFL number in dimension $d$ on a regular mesh is related to 1-D CFL number

$$
C F L_{d D}=\frac{1}{\sqrt{d}} C F L_{1 D}
$$

We see from table 1, (extracted from [5]). that the CFL number for GL points of order $r+1$ is merely the same as the CFL for Gauss points of order $r$. Moreover, if a leap frog scheme is chosen, the penalty terms can be centered whereas it is not possible for Gauss points in an explicit scheme.

The main advantage of Gauss points is to produce a more accurate integration (integration of the stiffness matrix is exact for straight elements) and consequently more accurate solutions. This increase of accuracy has been shown by a dispersion analysis [5].

Finally, both Gauss points and Gauss-Lobatto points have advantages, but the choice of which to use is difficult. Only experiments can provide guidance as to the best choice and then only if there is the possiblity of clear benefit. We show a comparison in the next section.

\section{Numerical results}

\subsection{Non-uniform flows}

Let us recall that Vector $U$ and matrices $A_{i}$ are then equal to :

$$
U=\left(\begin{array}{ll}
p \\
\rho_{0} & v_{1} \\
\rho_{0} & v_{2} \\
\rho_{0} & v_{3}
\end{array}\right) \quad A_{i}=\left(\begin{array}{cc}
v_{i} & c_{0}^{2} e_{i} \\
e_{i} & v_{i} \mathbb{I}
\end{array}\right)
$$

For the expression of boundary conditions in a discontinuous Galerkin framework, please refer to [4], where Neumann and first-order absorbing boundary conditions are developed. The computation of boundary integrals is performed with the same method as for the "jump" 
matrix.

The type of the time integration scheme is important, but not essential in this paper. So we have chosen to use an explicit, low-storage (2N) Runge-Kutta scheme for all the experiments [12]. For all the experiments, the meshes are produced by CUBIT, and isoparametric curved elements are used to correctly approximate the geometry.

\subsection{2-D computations}

The computational domain is a square $[0,12]^{2}$ with an interior circular hole. A Neumann condition is set on the circular hole, and boundaries $y=y_{\min }$ and $y=y_{\max }$. On the other boundaries, an absorbing boundary condition is set. In order to obtain a stationary flow compatible with the Neumann condition, one can solve the following Poisson problem

$$
\left\{\begin{array}{l}
\Delta u=0 \\
\frac{\partial u}{\partial n}=M \cdot n \text { on } \Sigma \\
\frac{\partial u}{\partial n}=0 \text { on } \Gamma
\end{array}\right.
$$

where $\Sigma$ represents the external boundary to the computational domain, and $\Gamma$ the interior boundaries. $M$ is the velocity vector of the flow at infinity and taken equal to :

$$
M=(0.5,0)
$$

The flow $v_{0}$ is then deduced from $u$ by the relation

$$
v_{0}=\nabla u
$$

Density $\rho_{0}$ and pressure $p_{0}$ are uniform so that the velocity $c_{0}$ is uniformly equal to 1 . The obtained flow around the disk is displayed in Fig 10. The source function $f$ is sinusoidal and is modulated by a gaussian function in time and in space (centered at the origin) as follows

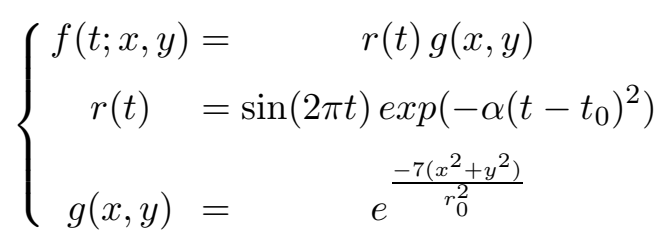

$r_{0}$ is the influence radius of the gaussian, and $\alpha=0.25$. The experiment is simulated until $t=15 \mathrm{~s}$. In table 2 , we compute the number of degrees of freedom and the number of operations needed to reach an error less than $2 \%$ for the solution at $t=14 \mathrm{~s}$. When referring to the number of degrees of freedom, multiply by the number of unknowns (three unknowns in 2-D) to get the size of a vector. Except for $Q_{2}$ and $Q_{3}$, the efficiency of the 

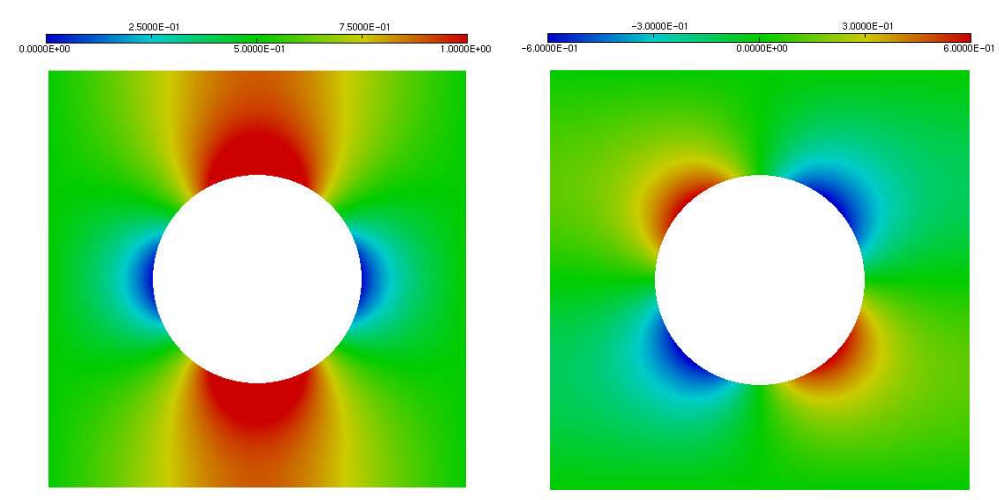

Fig. 10. Calculated flow around a disk. At left, component of the stationary flow along $x$, at right component along $y$.

\begin{tabular}{|c|ccccc|}
\hline Element & $Q_{2}$ & $Q_{3}$ & $Q_{4}$ & $Q_{5}$ & $Q_{6}$ \\
\hline \multirow{3}{*}{ Gauss } & 57987 & 29264 & 22275 & 18540 & 19649 \\
& $\Delta t=0.0138$ & $\Delta t=0.0173$ & $\Delta t=0.0168$ & $\Delta t=0.0167$ & $\Delta t=0.0145$ \\
& 69 GFlops & 27 GFlops & 21 GFlops & 18.2 GFlops & 23 GFlops \\
\hline Element & $Q_{3}$ & $Q_{4}$ & $Q_{5}$ & $Q_{6}$ & $Q_{7}$ \\
\hline \multirow{3}{*}{ Gauss-Lobatto } & 52016 & 31525 & 25416 & 23128 & 20608 \\
& $\Delta t,=0.0164$ & $\Delta t=0.0177$ & $\Delta t=0.0172$ & $\Delta t=0.0150$ & $\Delta t=0.0133$ \\
& 39.2 Gflops & 22.2 GFlops & 19.0 GFlops & 20.6 GFlops & 21.6 GFlops \\
\hline
\end{tabular}

Table 2. Number of dofs, time stepping and operations needed to reach an error less than $2 \%$ for the disk.

other orders shows little difference, thus there is no advantage between Gauss points and Gauss-Lobatto points.

Let us consider the flow around a NACA airfoil (see Fig 11). We see that near the two extremities of the airfoil, the flow is varying rapidly because of the high curvature. The airfoil geometry has been defined by a closed spline (the right end is curved, and is not a corner). Because the Neumann condition is imposed in a weak sense, this condition is not numerically satisfied, especially on the two ends of the airfoil. Hence, we need to modify the flow in order to satisfy this condition :

$$
v_{0 x} n_{x}+v_{0 y} n_{y}+v_{0 z} n_{z}=0
$$

The component modified is related to the maximum normal component. If this modification is not done, instabilities can appear. The computed solution is displayed in Fig 12. In this "difficult case", because of the sharp right extremity, Gauss-Lobatto points provide unstable algorithms, while Gauss points provide acceptable results. In table 3, we computed the 

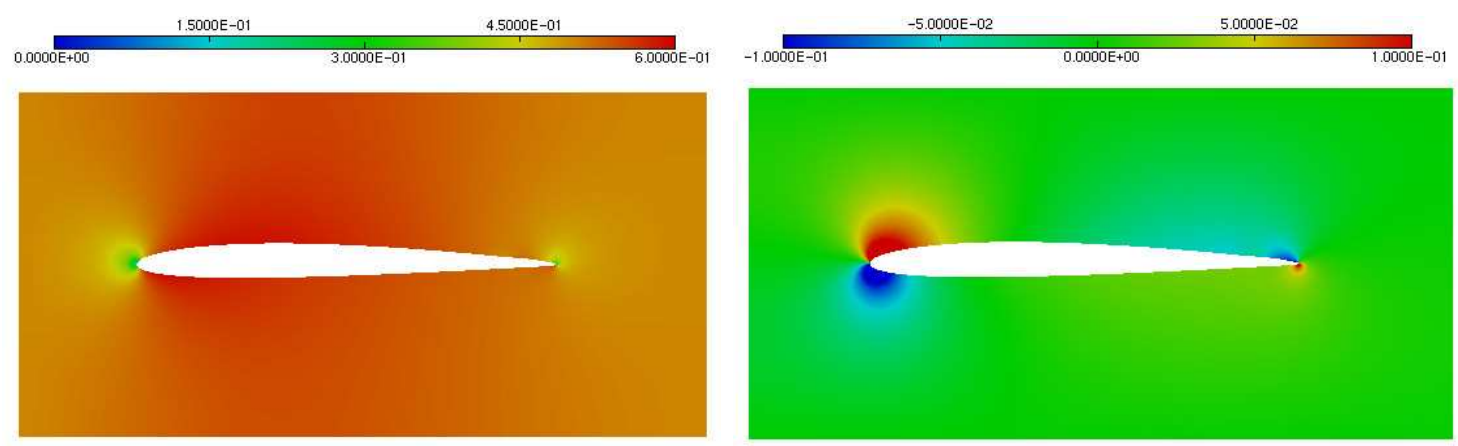

Fig. 11. At left, component of the stationary flow along $x$, at right component along $y$.
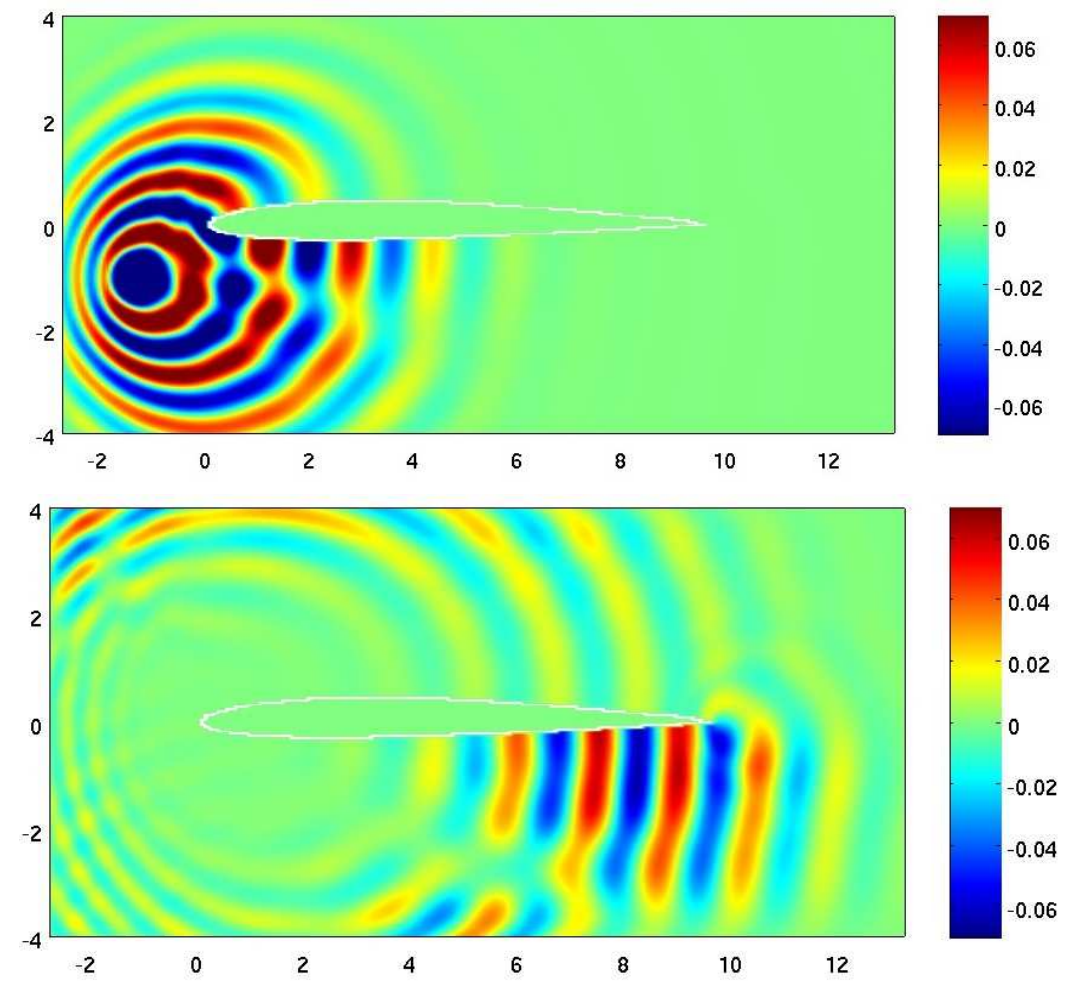

Fig. 12. Pressure at $t=9 \mathrm{~s}$ and $t=14 \mathrm{~s}$ for the NACA airfoil.

number of degrees of freedom and the number of operations needed to reach an error less than $2 \%$, for the solution at $t=14 s$. In that case, $Q_{3}$ is the optimal order, with 14.7 GFlops. Because of the geometry, local refinement near the extremities is needed for approximations of an order greater than three, reducing computational speeed.. The mesh used for $Q_{6}$ is displayed in the Fig 13. 


\begin{tabular}{|c|ccccc|}
\hline Order & $Q_{2}$ & $Q_{3}$ & $Q_{4}$ & $Q_{5}$ & $Q_{6}$ \\
Dofs & 27792 & 18000 & 15500 & 13788 & 14602 \\
Time Step & $\Delta t=0.025$ & $\Delta t=0.0195$ & $\Delta t=0.0139$ & $\Delta t=0.0134$ & 0.0085 \\
Operations & 18.3 GFlops & 14.7 GFlops & 17.9 GFlops & 16.9 GFlops & 29.1 GFlops \\
\hline
\end{tabular}

Table 3. Number of dofs, time stepping and operations needed to reach an error less than $2 \%$ for the NACA airfoil.

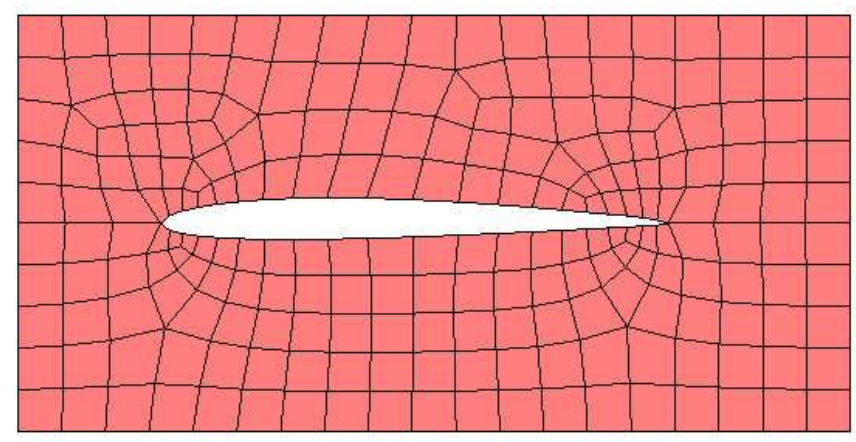

Fig. 13. Mesh used for $Q_{6}$ and NACA airfoil.

\subsection{3-D computations}

Another method to obtain a stationary flow, satisfying the condition $v \cdot n=0$ on the surface of the objects, is to solve a Poisson problem for $v_{0 x}$

$$
\left\{\begin{array}{l}
\Delta v_{0 x}=0 \\
v_{0 x}=0.5 \text { on } \Sigma \\
v_{0 x}=0 \text { on } \Gamma
\end{array}\right.
$$

where $\Sigma$ represents the external boundary to the computational domain, and $\Gamma$ the internal boundaries. The flow obtained for a spherical obstacle is displayed in Fig 14. On the sphere and the lateral sides of the external parallelipedic boundary, we impose a Neumann boundary condition. On the left and right side, a first-order absorbing boundary condition is set. Fig 15 shows the temporal evolution of the pressure. The temporal source is a sinusoid modulated by a gaussian function, like for the 2-D cases.

We will determine the minimal number of dofs needed to reach an error less than $5 \%$. The time step, dofs, and number of operations are displayed in table 4 . We observe a reduction by ten of the computational time, by using $Q_{7}$ instead of $Q_{3}$. We note that the complexity of Gauss points of order $r$ is almost equivalent to the complexity of Gauss-Lobatto points of order $r+1$. For example, $Q_{3}$ Gauss needs 31 TeraFlops while $Q_{4}$ Lobatto needs 26 Tflops and $Q_{4}$ Gauss needs 13.6 Tflops while $Q_{5}$ Lobatto needs 14.4 Tflops ... That is why the choice between the Gauss and Gauss-Lobatto points is not crucial. Nevertheless, we were not able to prove stability for Gauss-Lobatto points, and in practice such instabilities 


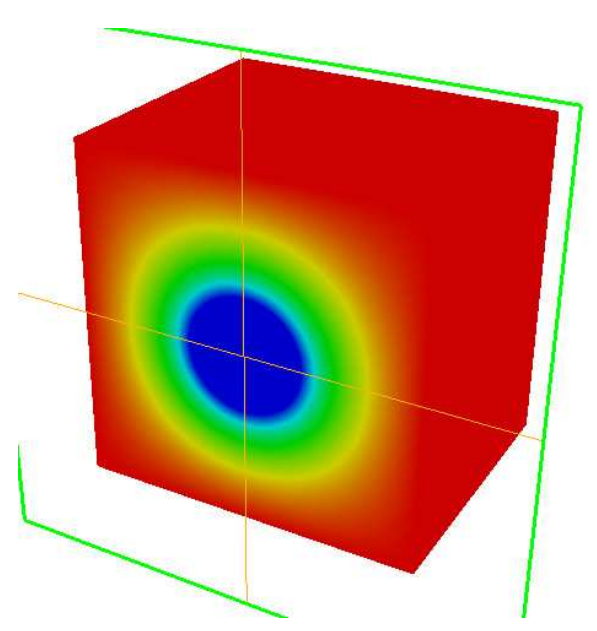

Fig. 14. Flow $v_{0 x}$ around the sphere.

\begin{tabular}{|c|ccccc|}
\hline Element & $Q_{3}$ & $Q_{4}$ & $Q_{5}$ & $Q_{6}$ & $Q_{7}$ \\
\hline \multirow{3}{*}{ Gauss } & 2.412 Mdof & 1.189 Mdof & 0.849 Mdof & 0.677 Mdof & 0.623 Mdof \\
& $\Delta t=0.0112$ & $\Delta t=0.0123$ & $\Delta t=0.0128$ & $\Delta t=0.011$ & $\Delta t=0.0111$ \\
& 31.23 TFlops & 13.55 TFlops & 9.22 TFlops & 8.61 TFlops & 7.98 TFlops \\
\hline \multirow{4}{*}{ Gauss-Lobatto } & 7.06 Mdof & 2.68 Mdof & 1.513 Mdof & 0.851 Mdof & 0.734 Mdof \\
& $\Delta t,=0.0105$ & $\Delta t=0.0119$ & $\Delta t=0.0121$ & $\Delta t=0.0129$ & $\Delta t=0.0124$ \\
& 81.3 Tflops & 26.16 TFlops & 14.39 TFlops & 7.64 TFlops & 6.99 TFlops \\
\hline
\end{tabular}

Table 4. Efficiency of the method for the sphere.

can be observed if we use this points for the unsplit formulation. A solution is to use the split formulation, which ensures the stability, whatever the quadrature formula. However, the split formulation induces additional computational time, that's why it seems better to use Gauss points, for which the stability (in the case of an uniform flow) is certified for the unsplit formulation. For non-uniform flows, we didn't observe any instability by using Gauss points with the unsplit formulation. 

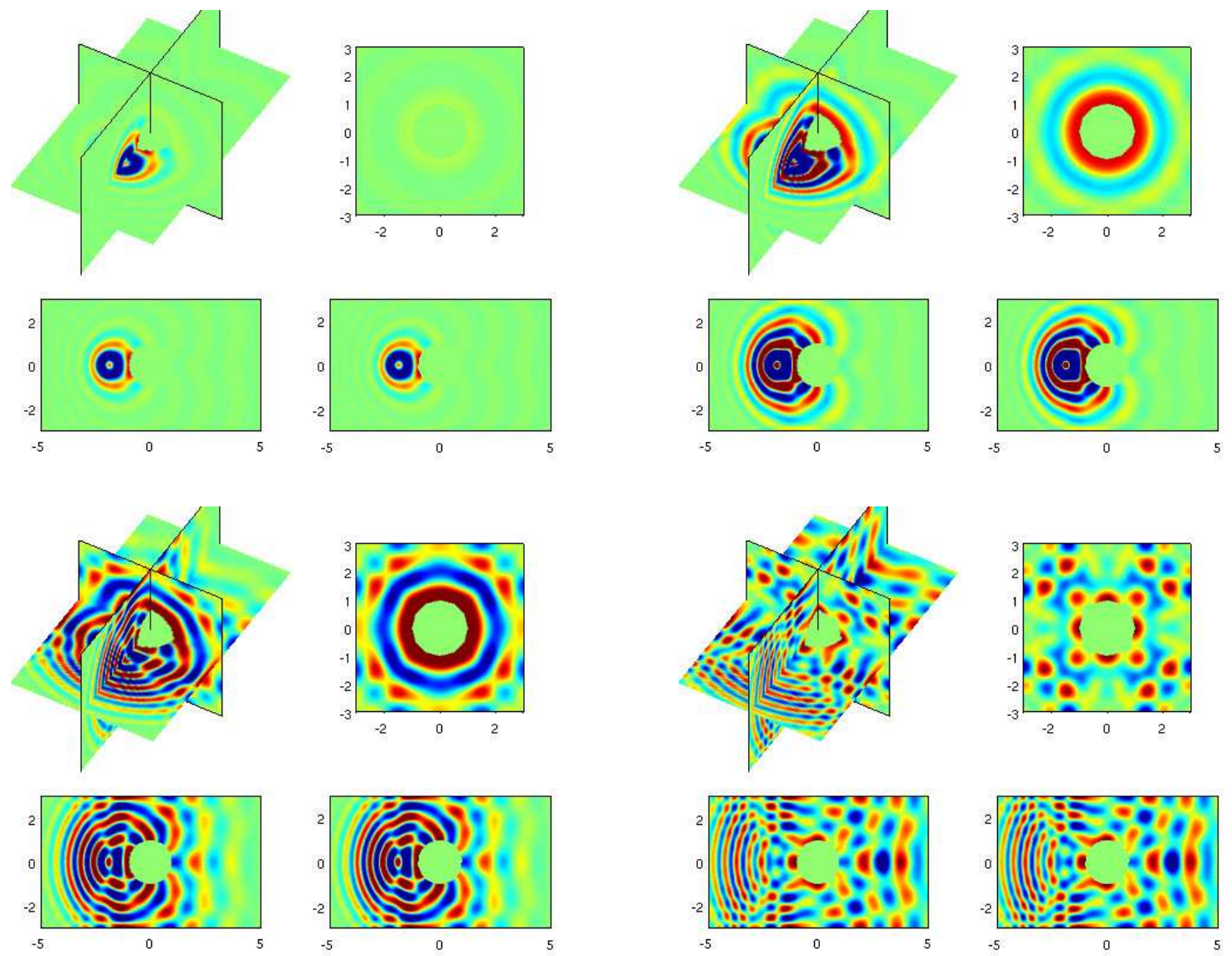

Fig. 15. Evolution of a gaussian source in space with a spherical obstacle. Snapshots at $t=6 s, 8 s, 10 \mathrm{~s}$ and $12 s$. 
[1] A-S Bonnet-Ben Dhia, E-M Duclairoir, G. Legendre and J-F Mercier, Time-harmonic acoustic propagation in the presence of a shear flow Journal of Computational and Applied Mathematics, 204(2), pp 428-439 (2007)

[2] M. Costabel and M. Dauge, Weighted regularization of Maxwell equations in polyhedral domains, Numer. Math. 93, pp 239-277 (2002)

[3] J.S. Hesthaven and T. Warburton, High-Order Nodal Methods on Unstructured Grids. I. Time-Domain Solution of Maxwell's Equations, J. Comput. Phys. 181(1), pp. 186-221 (2002)

[4] M. Bernacki, Méthodes de type Galerkin discontinu pour la propagation des ondes en aéroacoustique, PhD Thesis, Ecole Nationale des Ponts et Chaussées (2005)

[5] S. Pernet Etude de méthodes d'ordre élevé pour résoudre les équations de Maxwell dans le domaine temporel. Application à la détection et à la compatibilité électromagnétique., PhD Thesis, Université de Paris IX Dauphine (2004)

[6] P. Castillo, B. Cockburn, I. Perugia and D. Schoetzau, An a priori error analysis of the local discontinuous Galerkin method for elliptic problems, SIAM J. Numer. Anal. 38(5), pp. 1676-1706 (2000)

[7] P. Grob, M. Duruflé and P. Joly, Influence of the Gauss and Gauss-Lobatto quadrature rules on the accuracy of a quadrilateral finite element method in the time domain, submitted to Numerical Methods for Partial Differential Equations

[8] A. H. Stroud, Approximate calculation of multiple integrals, Prentice-Hall Inc., Englewood Cliffs, N.J. (1971)

[9] O. C. Zienkiewicz, The finite element method in engineering science, McGraw-Hill, London (1971)

[10] M. Duruflé, Intégration numérique et éléments finis d'ordre élevé appliqués aux équations de Maxwell en régime harmonique, $\mathrm{PhD}$ Thesis, Université de Paris IX Dauphine (2006)

[11] C. Bogey, C. Bailly and D. Juvé Computation of flow noise using source terms in linearized Euler's equations, AIAA Journal 40(2), pp. 225-243 (2002)

[12] M. H. Carpenter and C.A. Kennedy, A fourth-order $2 N$-storage Runge-Kutta scheme, NASA TM 109112, June (1994)

[13] G. Cohen, M. Duruflé Non Spurious Spectral-Like Element Methods for Maxwell's Equations, Journal of Computational Mathematics, vol 25, pp 282-304 (2007)

[14] G. Cohen, Higher-order Numerical Methods for Transient Wave Equations, Springer Verlag (2002)

[15] G. Cohen, X. Ferrieres and S. Pernet, A spatial high-order hexahedral discontinuous Galerkin method to solve Maxwell equations in time domain, Journal of Computational Physics, vol 217(2), pp 340-363 (2006)

[16] M. Bernacki and S. Piperno, A dissipation-free time-domain Discontinuous Galerkin method applied to three dimensional linearized Euler equations around a steady-state non-uniform inviscid flow, J. Computational Acoustics, vol. 14, No. 4, pp. 445-467 (2006) 